\title{
El Concepto de Naturaleza desde el Fisicalismo de Deutsch
}

The Concept of Nature from the Physicalism of Deutsch

Fabio Morandín Ahuerma*

[fabiomorandin.a@gmail.com]

\section{RESUMEN}

El presente artículo es una elucidación básica del concepto de naturaleza en relación con el hombre y la physis en tensión. Concluimos que la pregunta por la naturaleza imbrica por necesidad al Ser del hombre con implicaciones morales insalvables. A partir del andamiaje teórico y epistemológico que adoptemos, será el proyecto axiológico de sociedad.

\section{Palabras claves}

Ontología; Physis; Realidad; Hombre.

\section{ABSTRACT}

This paper is a basic elucidation of the concept of nature with relation to man and physis in tensile connection. In this paper we conclude that the question concerning nature necessarily imbricates man's being with insurmountable moral implications. Theoretical and methodological framework human beings adopt, determines their axiological project of society.

\section{KEYWORDS}

Ontology; Physis; Reality; Man.

\section{La naturaleza cómo creación del pensamiento humano}

Si el hombre —y sólo el hombre - es capaz de reunir en una noción el concepto griego de $\varphi u ́ \sigma \iota \varsigma$ (physis), ¿podríamos sostener que es únicamente una construcción intelectiva, más allá de lo fenoménico? ¿Puede una totalidad de lo real ser objetivada, contextualizada, organizada? ¿Acaso no existe un referente como totalidad, $\varphi$ ó ıৎ (naturaleza) más que en el plano de las ideas? Si otorgamos que la $\varphi u ́ \sigma \iota \varsigma$ es una parte de la cultura y no una entidad, se trataría de un constructo mental del ser humano, que además de ser utilizada, moldeada y explotada, es representada como una idea con tensión y poder. La naturaleza tiene un carácter de unidad, no forzosamente teológico-antropológico como creatio, pero aún esconde secretos sobre su estructura, por lo menos para el hombre. La pregunta nuevamente es: ¿Existe algo como la naturaleza o es un modo de concatenar en un concepto todo aquello que no ha sido hecho por el hombre?

\footnotetext{
* Postdoctorante en el Programa de Neuroética. Centro de Investigaciones Filosóficas (CIF), Buenos Aires, Argentina
} 
Las diferentes concepciones de naturaleza difieren entre las comunidades de conocimiento. Mientras que para unos ${ }^{1}$ la objetivación de la mirada a la naturaleza hace que ésta sea un referente hacia la trascendencia, para otros ${ }^{2}$ es una cosificación, un activo, propiedad privada explotable.

Al igual que el orden de la naturaleza, los antiguos chinos solían ver en el transcurso del tiempo una línea cíclica, infinita, interminable que, así como el día y la noche, el otoño que precede al invierno, el tiempo es un factor fundamental que se percibe por ciclos, la naturaleza atiende una orientación más allá de lo inmanente. Las imágenes de su arte, icónica y sagrada, representan la belleza, el orden y la perfección del cosmos. Cuanto más cerca se esté de la naturaleza, sostienen, ${ }^{3}$ más cerca se estará de la esencia del ser humano. Los caracteres ‘和’ representan la armonía entre naturaleza y humanidad, pero conllevan los conceptos de coexistencia pacífica, equilibrio, deber y por tanto, respeto.

Lo anterior contrasta con la visión occidental de que la biósfera le pertenece al hombre y que, por tanto, está a su servicio para ser explotada. El enfoque de que la naturaleza está ligada a la economía hace de ésta una fuente inagotable de materias primas para la industrialización de productos de consumo ${ }^{4}$ En un contexto utilitario se tiene la idea de que la naturaleza está ligada al concepto de economía.

Desde la Modernidad podemos presenciar una distinción sujeto-objeto que se traduce entre hombre-naturaleza. Lo humano como algo objetivado en lo que se llamó recursos naturales bajo la égida de la racionalidad humana. "El principio de lo real es algo de tipo conceptual, al alcance de la inteligencia humana y manipulable a través de las teorías científicas y las prácticas tecnológicas". 5 Esto, por supuesto, debe ser analizado en tanto el hombre encuentra un referente mediato en su inteligencia instrumental en la biósfera.

En la cultura de mercado el ser humano tiene la potestad para hacer de la naturaleza su dominio. El apoderarse de ella es la parte más alta de la cadena, en que el valor de la mercancía está por encima de todos los seres vivos, y por tanto, le da las facultades para su utilización con fines de explotación. Esta forma de ver al mundo y a la naturaleza hace que el ser humano se conciba así mismo como el ápice de esta cadena.

La vida silvestre, la biósfera y la realidad es de nadie, está ahí para ser aprovechada de la mejor manera posible, como una muestra de racionalidad irracional. No importa que la explotación de los recursos naturales ponga en peligro la subsistencia de las nuevas generaciones y la viabilidad de la vida misma, ya que este es un efecto colateral solo probable y de largo plazo, se arguye. ${ }^{6}$

El ser humano tiene un sentido inmediato de la propiedad sobre la naturaleza. El hombre hace uso eficaz y eficiente, desde su racionalidad instrumental para conseguir sus fines no solo de subsistencia sino de generación de riqueza. Queda de este modo fuera del marco teórico la idea de sustentabilidad, entendida como: "Desarrollo que satisface las necesidades del presente sin comprometer la capacidad de las generaciones futuras para satisfacer sus propias necesidades". ${ }^{7}$ Cuando se habla de control nos referimos al control de la naturaleza para cubrir las necesidades básicas y no básicas del ser humano, pero también al control simbólico o fáctico. Existe autoridad informal en ello, una capacidad de presión y tensión en la que la naturaleza se encuentra amenazada por el hombre.

Sin embargo debemos hacer el contraste entre el poder de la naturaleza -y este es un ítem de análisis - que está por encima, y por mucho, del poder de control que el ser humano tiene sobre su entorno. Hay una necesidad por controlar la naturaleza, vista ésta como un conjunto de elementos con cierta fuerza; la naturaleza nos puede ser hostil, en todo momento podemos ser superados y lo que el hombre hace es, precisamente, creer dominar esa naturaleza, antropologizarla de modo tal que quede

\footnotetext{
${ }^{1}$ Artigas, M. La inteligibilidad de la naturaleza (Pamplona: Eunsa, 1995).

${ }^{2}$ Martínez, J. y Schlupman, K. La ecología y la economía (México: Fondo de Cultura Económica, 1991).

${ }^{3}$ Zhuang Zi. Maestro Chuang Tsé (Barcelona: Kairós, 1992), 27.

${ }^{4}$ Boff, L. La ecología como nuevo espacio de lo sagrado (Madrid: Trotta, 1996), 95.

${ }^{5}$ Hernánz, J. "La relación hombre/naturaleza como entorno construido". En Ciencia y el Hombre 19, Universidad Veracruzana, México, (2006) 19 (3) http://bit.ly/1GfuiQB

${ }^{6}$ Martínez y Schlupman, 1991, 77.

? Komiyama, H. y Takeuchi, K. "Sustainability science: building a new discipline". En Sustainability Science 1-6, (2006), 3.
} 
bajo su control; aunque en muchos casos salga del ámbito que el hombre tiene. ${ }^{8}$ Basta ver la verdadera dimensión del hombre frente a los fenómenos climatológicos de la naturaleza: tsunamis, tornados, terremotos, etc. para comprobar una y otra vez su indefensión.

La naturaleza es, en el fondo, un problema interdisciplinario: de tipo moral, político y ético; y es la construcción de este mundo humano, hecho a expensas del mundo natural, el que nos enfrenta con disyuntivas morales insalvables.

Es la concepción de la vida. No es ociosa la pregunta filosófica: ¿Cuál es la diferencia entre algo vivo y algo muerto? Todo lo real tiene poder y la condición de poder es la vida misma. Está omnipresente en cada rincón de la realidad pero las explicaciones mecanicistas, físico-químicas parecen no ser suficientes para explicarla ¿Qué es eso que llamamos vida como parte inherente de la naturaleza? ${ }^{9}$

Si bien la biología molecular ha estado a la punta de una definición más precisa de vida, sobre todo a partir del descubrimiento del ácido desoxirribonucleico o ADN, lo cierto es que muchos científicos no quieren arriesgarse siquiera a dar una definición precisa. Coinciden en que hay tres propiedades que podrían ser necesarias y que juntas son suficientes para que exista vida pero, no hay una descripción concluyente. Las características son: auto reproducción, evolución y metabolismo. ${ }^{10}$

A partir de la definición de vida se hacen otras distinciones sobre lo que está vivo y lo que no lo está. La ciencia actual no ha sido capaz de crear siquiera una célula. Los equipos de avanzada en los laboratorios de biología sintética han hecho promesas sobre la replicabilidad de una célula viva morfológica, funcional y autopoyética, ${ }^{11}$ pero hasta el momento no han logrado la creación, propiamente dicha, de ningún tipo de vida.

Si se tiene el poder de transformar la biosfera, de enviar cohetes al espacio, de hacer represas que cambian el cauce de caudalosos afluentes, o de construir bombas lo suficientemente poderosas como para borrar de la faz de la tierra a ciudades enteras, ¿por qué el hombre no ha sido capaz de darle vida a algo tan insignificante como una bacteria?

Por un lado sentimos que la vida nos pertenece, que es algo que poseemos y que está intrínsecamente relacionado conmigo mismo, sin embargo, por otro lado, la vida no nos pertenece porque es algo dado desde fuera, es algo producido más allá de la esfera de nuestra propia voluntad.

En la posición mecanicista del filósofo y científico David Elieser Deutsch no cabría que la vida esté dada "desde fuera" pues, además de un conjunto de funciones moleculares químicas, no existe algo añadido. Para él lo que está presente en aquello que manifiesta tener vida son códigos genéticos escritos como secuencias de los símbolos A, C, G y T que corresponden a los nombres de las moléculas componentes (adenina, citosina, guanina y tiamina) y su característica más importante es la replicabilidad. ${ }^{12}$ Eso es todo.

Sin embargo, no deja de resultar llamativo que las nuevas propuestas entre sapienciales y filosóficas que está produciendo la época moderna, lo que hacen es un rescate hacia lo sagrado y hacia el mundo religioso; no necesariamente hacia un dios del modo monoteísta pero tiene relación intrínseca con una dimensión ulterior. ${ }^{13}$

El concepto de Dios en lo Natural no es un dios antropologizado, se trata de un principio fundante de la vida personal que cada uno construye bajo un fundamento 0 sin este. La figura del fundamento que el hombre va construyendo en su propia realidad, por el hecho de vivir, puede ser el dios real que se

\footnotetext{
${ }^{8}$ Hernanz, J. "Una propuesta iberoamericana para la superación de la filosofía moderna: Zubiri y la metafísica de la nuda realidad". En Revista Estudios Latinoamericanos, 11, Universidad de Nariño (2011) 20-21. http://bit.ly/1IThbuW y Cfr. Maritain, J. Filosofía de la naturaleza (Buenos Aires: Club de lectores, 1947), 57.

${ }^{9}$ Carroll, W. y Baldner, S. Aquinas on Creation (Toronto: Pontifical Institute for Mediaeval Studies, 1997), 6.

${ }^{10}$ Machery, E. "Why I stopped worrying about the definition of life and why you should as well". En Synthese, 185, 1 (2012) 145-164.

${ }^{11}$ Craig, J. La vida a la velocidad de la luz (Madrid: Crítica, 2013), 11-26.

${ }^{12}$ Deutsch, D. The Fabric of Reality. The Science of Parallel Universes and Its Implications (New York: Penguin, 1997) 119. Tr. español: La estructura de la realidad. (Barcelona: Anagrama, 1999).

13 Jaki, S. Cosmos and Creator (Chicago: Regnery Gateway, 1980), 22.
} 
construya, ya sea ùna visión teísta, agnóstica 0 atea, no consiste primariamente en la aceptación 0 negación conceptiva de su existencia como término de un razonamiento.$^{14}$

¿Cómo dejar de lado la parte espiritual de la vida sin caer en el reduccionismo mecanicista que equipara la vida a la mera unión de células (físico-química) y que la define como un estado o carácter especial de la materia alcanzado por estructuras moleculares específicas, con capacidad para desarrollarse, mantenerse en un ambiente, reconocer y responder a estímulos y reproducirse permitiendo la continuidad? ${ }^{15}$ Esa es una definición descriptiva de los organismos vivos pero no nos dice qué es propiamente la vida.

\section{Apxń y lo real}

La única forma de dominar lo real en la naturaleza es si se capta el $\alpha \rho x \eta ́$ (arkhé), traducido como comienzo, fuente, principio u origen, comienzo del universo o el primer elemento de todas las cosas; si se insiste, sustancia, es decir, aquello que no necesita de ninguna otra cosa para existir. El $\alpha \rho x n ́$ es un concepto insertado en el logos como un concepto mítico, no es una deidad, no es un ser creador, es un principio explicativo, no creativo, del porqué todo lo real está unificado.

Aristóteles en la Metafísica $(1,3)^{16}$ explica que los presocráticos consideraron los primeros principios pero desde el punto de vista de la propia materia. El principio de todos los seres es aquello de donde salen todos, de donde proviene todo lo que se produce, y adónde va a parar toda destrucción. La sustancia misma persiste bajo sus diversas modificaciones.

Hay una totalidad del todo que tiene que ver con el Пoxń y esto nos lleva a una doble distinción, la primera: hombre-naturaleza; la segunda: mundo natural vivo-mundo natural físico. Son dicotomías que nos exigen cierto rompimiento para construir nuevas líneas argumentativas. Somos reales porque estamos en un ambiente que gestionamos, que somos capaces de transformar para tener un mundo cada vez más humano y, en la medida en que nos alejamos de lo natural nos volvemos más sofisticados y sapientes. Pero al parecer con insensibilidad para reconocer el milagro que representa la vida. ${ }^{17}$ Esto tiene por supuesto implicaciones objetivas.

Un problema de tipo moral, político y ético, sobre todo de los últimos dos siglos, es la construcción de este mundo humano que está hecho a expensas del mundo natural. ${ }^{18}$ No solo porque lo explotamos sino porque, para hacernos más racionales, existe un imperativo de que debemos separarnos cada vez más de la naturaleza; la polis, y no en el sentido griego sino en el sentido de urbe, técnicamente es el lugar donde nos hacemos seres humanos y esto debe estar separado del mundo natural. ${ }^{19}$

¿Cómo entramos en la naturaleza? ¿Cómo nos vemos en su espejo? El rompimiento de la dicotomía sujeto-objeto es visible cuando el hombre ha quedado disuelto en una magnitud que puede existir sin la presencia de la naturaleza. El mundo anthropo-óntico es una visión cómoda porque nos disponemos como centros del universo. Un fin para sí.

La cultura occidental parte de un supuesto: no es posible inteligir totalmente la estructura de la realidad. No puedo tener la experiencia de la estructura de la realidad. Lo único que se puede tratar de aprehender son sus fenómenos, como por ejemplo el llamado cambio climático ${ }^{20}$ Se vive en el mundo natural y se puede tener una idea de lo que son las cosas o determinar qué se puede hacer, qué plantas se pueden comer, cuáles no. Pero esta experiencia empírica no supone una captación de lo real. Aquí

\footnotetext{
${ }^{14}$ Hernánz, 2011, 17.

${ }^{15}$ Cfr. Deutsch, 1997, 197 y Craig, 2013, 46.

${ }^{16}$ Aristóteles, Metafísica (Madrid: Gredos, 2007).

${ }^{17}$ Cfr. Jaki, 1980, 26-35.

${ }^{18}$ Komiyama y Takeuchi, 2006, 14.

${ }^{19}$ Cfr. Boff, 1996, 99.

${ }^{20}$ Cohen, S., Demeritt, D., Robinson, J., \& Rothman, 'D. "Climate change and sustainable development: towards dialogue”. En Global Environmental Change 8 (4), (1998) 341-371.
} 
está el gran núcleo de la posibilidad de la filosofía porque solo a través de ella existe la posibilidad de captar la unidad de lo real.

Para Deutsch son las teorías científicas las únicas capaces de dar una explicación causal de la realidad, tal como advierte en su obra The Fabric of Reality:

Scientific theories explain the objects and phenomena of our experience in terms of an underlying reality which we do not experience directly. But the ability of a theory to explain what we experience is not its most valuable attribute. Its most valuable attribute is that it explains the fabric of reality itself. As we shall see, one of the most valuable, significant and also useful attributes of human thought generally is its ability to reveal and explain the fabric of reality. ${ }^{21}$

Aquí hay un salto de lo que empíricamente se puede captar de lo real y lo que empíricamente es la estructura de esa realidad, y el carácter trascendente de ésta. La posición de la ciencia es pensar que se puede aprehender la realidad, sin más. No se habla aquí de una metaconstrucción de un sentido de lo real, sino de lo real como la idea más general después del Ser.

¿Cómo puedo transitar desde las leyes científicas hasta una cosmovisión total de la realidad? Lo real es algo unitario que puede darse y sostenerse como existencia. La mejor manera de ver la realidad para la ciencia, por ejemplo, es verla en un lenguaje axiomático con matices.

Lo real es algo unitario y esa unidad se puede traducir en una unidad del cosmos que puede expresarse a través del lenguaje matemático. Esta es una aseveración que refleja una aspiración de la comunidad de conocimiento. El reto, suponen, ${ }^{22}$ es tratar de hacer una teoría onmiabarcadora del cosmos. No se puede expresar de forma racional, simple, la estructura total de la realidad. Incluso el propio Stephen Hawking ${ }^{23}$ ha declarado haber abandonado su pretensión original de construir una "teoría del todo".

No estamos hablando de fuerza sino de campo. La ciencia trabaja a través de símiles como la llamada teoría del caos que lejos de afirmar que todo es caótico y azaroso apunta a que existe cierta estructura y orden dentro de ese caos. En la mayoría de los sistemas clásicos el caos es la inestabilidad del movimiento y una diferencia, así sea ligera, entre dos estados iniciales que puede resultar en desviaciones crecientes entre las dos trayectorias resultantes exponencialmente.

La definición de caos que Deutsch propone es la siguiente:

The instability in the motion of most classical systems. A small difference between two initial states gives rise to exponentially growing deviations between the two resulting trajectories. But reality obeys quantum and not classical physics. Unpredictability caused by chaos is in general swamped by quantum indeterminacy caused by identical universes becoming different. ${ }^{24}$

Todo lo real, aunque caótico, tiende a ordenarse. Este ordenamiento al parecer no obedece a una regla predeterminada. No hay un motivo que haga que las cosas sean siempre $X$ entonces $Y$, como condición necesaria, así y sólo así. Pero aún con ello, no parece que se rompa la racionalidad del caos.

\footnotetext{
${ }^{21} \mathrm{Tr}$. "Las teorías científicas explican los objetos y fenómenos que conocemos por medio de la experiencia en términos de una realidad subyacente que no experimentamos de modo directo. Pero la capacidad de una teoría para explicar lo que conocemos solo por la experiencia no es su más valioso atributo, sino el hecho de que explique la estructura de la realidad. Como veremos, uno de los atributos más valiosos y significativos, así como útiles, del pensamiento humano, en general, es su capacidad para descubrir y explicar la estructura de la realidad”. Deutsch, 1999, 8.

${ }^{22}$ Deutsch, 1997, 229-266.

${ }^{23}$ Hawking, S. "Gödel and the end of physics". http://www. hawking.org.uk/index.php/lectures/91 (2002).

24 "Caos. Inestabilidad del movimiento en la mayoría de los sistemas clásicos. Una ligera diferencia entre dos estados iniciales origina desviaciones exponencialmente crecientes entre las dos trayectorias resultantes. Pero la realidad obedece a las leyes de la física cuántica, no de la clásica. Normalmente, la impredecibilidad provocada por el caos es absorbida por la indeterminación causada por el proceso mediante el cual universos idénticos se vuelven diferentes” Deutsch, $1999,247$.
} 
Ha sido construido de formas muy diversas y este universo tiene una multiplicidad de reglas, pero existe una que no permite que se colapsen entre sí. Existe una coherencia que permite no contradecirse, se observa una identidad sutil con diferencias internas.

Lo real tiende a organizarse de tal manera que haya diversidad en lo complejo dentro de una unidad. El concepto de ley vinculado a la naturaleza sigue suponiendo que existe una racionalidad que se impone sobre cualquier cosa existente, ya sea divina o no, y de hecho podemos ver que la naturaleza impone leyes innegables. ${ }^{25}$

Ahora contrastémoslo con otro argumento que, si lo analizamos, afirma de algún modo lo antes expuesto. A lo largo de la historia el hombre ha buscado certidumbre a través de sus propias inducciones, esto es, de la observación repetida de objetos o acontecimientos de la misma índole y trata de establecer conclusiones para aquellos que comparten las mismas condiciones. Así, establece cierto tipo de generalización como un razonamiento inductivo básico, sin embargo se afirma que ese salto cognoscitivo genera un problema, el llamado problema de la inducción ${ }^{26}$ en el que no me detendré pero baste citarlo. ${ }^{27}$

Aunque no estamos seguros de que realmente exista tal problema, Deutsch considera que la inducción si bien no puede ser justificada como método para la apropiación de conocimiento, tiene una pretensión clara de objetividad, llámese como se llame al poder de hacer generalizaciones:

What we need is an explanation-centred theory of knowledge: a theory of how explanations come into being and how they are justified; a theory of how, why and when we should allow our perceptions to change our world-view. Once we have such a theory, we need no separate theory of predictions. For, given an explanation of some observable phenomenon, it is no mystery how one obtains predictions. And if one has justified an explanation, then any predictions derived from that explanation are automatically justified too. ${ }^{28}$

Además de la ciencia y la filosofía existen otros vínculos con otras expresiones culturales que podríamos valorar, interrelaciones con otras disciplinas, por ejemplo el arte y la religión. La religión es un campo en que la naturaleza tiene una función sustentante porque antes de la sacralización antropológica, hay una sacralización primigenia de la naturaleza como en algunas culturas orientales y américoprecolombinas. Asimismo el arte es, como se ha dicho muchas veces, lo más cercano a lo sacro. La representación del hombre de su dimensión trascendente a través de la creación. Es el momento en que el hombre se equipara a ese ser creador, la divinidad y que puede, de muchas maneras, expresar lo que de suyo $^{29}$ es naturaleza, entendida como la multiplicidad de lo real. ${ }^{30}$

Hay una separación no resuelta entre arte-religión y naturaleza. Todo el arte figurativo es una demostración de lo divino en la naturaleza. ${ }^{31}$ Los pintores y artistas desde el siglo VII hasta el XVI buscan

\footnotetext{
${ }^{25}$ Cfr. Artigas, 1994, 223-226. inferencia nueva, no prueba que para lo futuro continuará así" Hume, 1980, 42. toda predicción derivada de ella lo está también de manera automática" (Deutsch, 1999, 48).

${ }^{29}$ Zubiri, J. Sobre la realidad (Madrid: Alianza Editorial, 1966) 34.

${ }^{30}$ Cfr. Maritain, 1947, 11-21.

${ }^{31}$ Cfr. Aubert, J. Filosofía de la naturaleza (Barcelona: Herder, 2001), 56.
}

${ }^{26}$ Hume, D. (1980) Investigación sobre el conocimiento humano (Madrid: Alianza, 1980), 42.

${ }_{27}$ Todas las inferencias sacadas de la experiencia suponen, como su fundamento, que el futuro semejará al pasado y que los poderes similares se unirán a similares cualidades sensibles. Si existiera alguna sospecha de que el curso de la naturaleza pudiera cambiar y que el pasado no sirviera de regla para el futuro, toda experiencia resultaría inútil y no podría dar origen a inferencia 0 conclusión alguna. Por lo tanto, es imposible que argumentos sacados de-la experiencia puedan probar la semejanza del pasado con el futuro, ya que todos los argumentos semejantes están fundados en la suposición de tal semejanza. Aun admitiendo que el curso de las cosas siempre ha sido regular, este solo hecho, sin ningún argumento 0

28 "Se requiere de una teoría que permita comprender cómo se producen y se justifican las explicaciones; una teoría que permita comprender cómo, por qué y cuándo debemos aceptar que nuestras percepciones cambien nuestra concepción del mundo. Una vez la tengamos, no necesitaremos ya separar la teoría de las predicciones, puesto que, si se tiene la explicación de un fenómeno observable, hacer predicciones acerca de él no es ningún misterio. Y, una vez justificada una explicación, 
representar lo sacro en sus obras. Estaban convencidos, sobre todo en la Edad Media, de que la divinidad no es una creencia, es un referente obligado del arte como la única concepción objetivada de lo que está allende a sus 0jos. ${ }^{32}$

Aún en la iconografía laica encontramos una sublimación del espíritu y de las necesidades intelectivamente profundas de los artistas. Se convierten en el oráculo. La religión ha tenido también una forma de diálogo y panegírico en las diversas formas de arte, una encriptada en la otra. Más adelante con el arte no figurativo la comunicación del artista con el medio es más importante que la creación misma, sin embargo el acto de creación sigue siendo por naturaleza el mismo. ${ }^{33}$ La búsqueda por alcanzar nuevos horizontes sobre lo dado, ir a un más allá en que las reglas se rompan y aflore, no las sombras, sino la realidad tal como es en el sentido de la comprensión ideal. La naturaleza es lo otro, pero otro con capacidad y fortaleza. La vida es un principio de acción y es, ante todo, algo sacralizado. La idea de naturaleza que tengamos depende de cómo articulemos estas cuatro dimensiones: la concepción filosófica, la religiosa, la estética 0 artística y, por supuesto, la científica.

El fenómeno trascendental, entre todos los fenómenos, es la vida. Ténemos un nudo que debemos resolver, la visión antropológica es seguir el camino de la ciencia. El punto de quiebra es una teoría del conocimiento que dé una explicación no racional del cosmos porque, de cualquier modo, todo parece indicar que la racionalidad tiene límites precisos en esta comprensión del universo macro. ${ }^{34} \mathrm{El}$ entendimiento humano, simplemente, no alcanza para abarcarlo.

Si bien podemos conceder que la naturaleza es una construcción antropológica y cultural, el gran problema del conocimiento sigue siendo la naturaleza misma como un todo. El problema ontológico es también un problema epistemológico ¿Qué condiciones tenemos del conocer? ¿Cómo se configura lo humano frente a lo otro natural que autorefiere al propio hombre? El problema de la realidad entendida como subjetividad es la realidad de un tipo de representación del sujeto.$^{35}$ No significa que sea una idea azarosa 0 un capricho intelectual, pero es una construcción antropológica.

Entonces la naturaleza es un producto de la cultura hecha por el hombre, la dimensión moral que ella implica tiene de facto un compromiso axiológico sobre lo que debería ser y lo que implica el misterio de la vida.

\section{A manera de conclusión}

La unidad de lo real, la dicotomía entre lo extraordinariamente diverso de la realidad y la unidad que detrás de ella puede descubrirse nos da la pauta para argumentar en favor de un sentido ontológico de la naturaleza como totalidad de la realidad. Existe una serie de estructuras en el nivel microscópico y en el nivel cósmico que, desde lo más elemental hasta las formas más complejas, encierran una ingeniería que el ser humano puede sólo parcialmente observar y menos aún comprender en su totalidad. Existe una complejidad en la realidad y en los elementos que la conforman que no es posible abarcar en su totalidad, al menos a través de una racionalidad teórica o instrumental.

La intelección de lo concreto es mediada a través de instrumentos de observación y de medición de fenómenos que no hacen otra cosa que acercarnos a los elementos constitutivos de estos, pero que en realidad no logran ser aprehendidos por el sujeto. Romper con esta dicotomía sujeto-objeto es posible en la medida en que veamos a la realidad como un todo (aunque suene contradictorio) constituido por micro y nanocosmos, aun así seguimos sin poder entrar en ella para duplicarla.

Cuánto más se descubre los secretos que guardan los fenómenos naturales y la materia en su conjunto, aparecen más puntos de divergencia en cuanto a aquello de lo que están hechas la materia inerte y la materia viva. Desde la célula hasta los planetas distantes se descubre que todo está

\footnotetext{
${ }^{32}$ Cfr. Trusted, J. Physics and Metaphysics (London: Routledge, 1991), 48.

${ }^{33}$ Wallace, W. "The Intelligibility of Nature: A Neo-Aristotelian View". En The Review of Metaphysics 38 (1984) 33-56 y Carroll y Baldner, 1997, 8.

${ }^{34}$ Aubert, 2001, 22-34.

${ }^{35}$ Wallace, 1986, 38.
} 
conformado por una misma naturaleza, los mismos elementos que están bajo los efectos de los mismos códigos.

Por paradójico que parezca, las teorías del caos han demostrado que detrás del desorden existe cierta coherencia, que le da unidad a lo diverso. En el fondo se cumplen patrones, una y otra vez, que demuestran la existencia de una red de relaciones que se expresan en la materia, en la fuerza y en el tiempo. Contrariando lo afirmado por Hume y el supuesto problema de la inducción.

No percibimos la realidad como una fatalidad, se trata, como advirtió Zubiri ${ }^{36}$ de una inteligencia sentiente que de algún modo nos permite captar aquello que de suyo está detrás de la realidad o en ella. No se trata únicamente de una impresión sensorial que puede engañarnos o que depende del sujeto que la perciba. Cada ojo tiene una visión diferente y no que el mundo sea diferente cada vez que lo veamos. Hay una realidad que le es inconmensurable y que no puede ser cambiada de acuerdo con el sujeto que la perciba. No se afirma con esto un realismo inocente. El ser humano es el único ser capaz de captar esa realidad peculiar de manera inteligente, abstraer, sintetizar, analizar y sacar conclusiones de esas impresiones, aun cuando no pueda abarcar, ni siquiera inteligir, su totalidad.

Por eso en esta impresión de realidad se actualiza lo presente en una impresión que tiene una verdad real. Toda realidad es trascendentalmente en su constitución una interioridad y una exterioridad. Es una interioridad que se manifiesta en la exterioridad entendida como su propia dimensión y es ella a la que podemos acceder, medir, dividir y volver a unir. ${ }^{37}$

La naturaleza guarda una interioridad fáctica que descubrimos a través de los fenómenos y la inteligencia sentiente. Es ahí donde gravita la dimensión epistemológica del ser y del conocer. Para nosotros ese es el sentido ontológico del problema de la naturaleza y de su ser. No es el problema del ser sino del conocimiento, un estar ahí, digámoslo así, trascendental.

La existencia es el sentido de la vida para el ser sentiente que somos. Para la naturaleza por su propio carácter constitutivo tiene una esencia cerrada, no así para el nosotros, que en medio de la calidad de posibilidad requerimos caminar por una ruta única y personal de sentido.

El valor intrínseco de la vida y del sujeto que la posee debe ser entendido como parte sustancial a su constitución. La propuesta aquí es romper con la duplicidad entre materia y vida, una oposición que divide a la naturaleza entre lo animado y lo inanimado, cuando todo cuanto existe en el universo tiene, de alguna forma, una característica dada desde fuera, creada que contempla la diferencia ontológica entre el ser y el no-ser.

La vida, especialmente la vida humana, tiene por lo menos tres dimensiones: Una dimensión individual, compuesto físico-psíquico, que consiste en la pertinencia de la existencia y en la subsistencia del cuerpo manteniéndolo sano. Una segunda dimensión que tiene que ver con lo antropológico y lo social, en las relaciones que el hombre genera en relación con los demás, en relación con el mundo físico que lo rodea y en donde se enmarca la parte moral de su relación con la naturaleza.

Y finalmente una dimensión más allá de lo físico como ente trascendental, en un mundo que, si encontramos el valor sagrado de la vida, ese valor, como dijimos antes, está dado desde fuera, por lo que la vida adquiere una dimensión sacra que de suyo tiene un valor intrínseco como única posibilidad de trascender entre lo real intrínseco y lo trascendente. ${ }^{38}$

Regresemos a la pregunta: ¿Cuál es el lugar del hombre en el cosmos? ¿Qué papel tiene la naturaleza humana en la realidad? Hay una preocupación cultural, psicológica y filosófica, incluso existencial, sobre el sentido de la vida y del ser humano. Entonces, ¿qué somos en la realidad? Tenemos la esfera de la realidad y la naturaleza, estamos en ella, la percibimos, la tocamos pero dentro de ella existen interrogantes indisolubles.

La naturaleza tiene un sentido antropológico. La naturaleza no son precisamente los árboles, los ríos, los animales, tampoco es una construcción teórica, sino una construcción pragmática de lo que el hombre concibe a su alrededor. La naturaleza también es una proyección moral del hombre. Nuestra idea

\footnotetext{
${ }^{36}$ Zubiri, 1962, 40-45.

${ }^{37}$ Cfr. Zubiri y Apalátegui, X. Sobre la esencia (Madrid: Alianza Editorial, 1962), 34.

${ }_{38}$ Zubiri, 1966, 111.
} 
de la naturaleza es el espejo donde nos miramos y aquello frente a lo que nos contraponemos sin darnos a veces cuenta de que estamos inmersos y que dependemos literalmente de ella.

Hasta que logremos compartir esta visión y sigamos actuando como el ápice del universo, la lógica instrumental de los recursos naturales y su explotación seguirá impactando de manera negativa la sustentabilidad del planeta. Por ejemplo, como advierte Weisman, ${ }^{39}$ los plásticos, especialmente los de tereftalato de polietileno, las pilas y las botellas de vidrio, podrían ser nuestro legado más duradero. Las soluciones deben ser radicales y no se necesita llegar a depender del fantasma de nuestra desaparición como especie para tomar decisiones definitivas y de largo plazo.

${ }^{39}$ Weisman, A. The World Without Us (New York: Thomas Dunne Books, 2007), 86. 